Patricia Stupariu*

José Alonso Olmedo*

María Isabel Cambón Murcia**

\title{
EVOLUCIÓN DE LAS CONEXIONES ENTRE BANCOS Y SECTORES FINANCIEROS \\ NO BANCARIOS EN EL SISTEMA \\ FINANCIERO ESPAÑOL
}

Este trabajo describe la evolución de las interconexiones dentro del sistema financiero español (dimensión transversal del riesgo sistémico) distinguiendo las conexiones directas y las indirectas. El análisis concluye que las interconexiones directas del sector bancario con los otros sectores son reducidas. Respecto a las indirectas, la relevancia de los sectores no bancarios ha venido aumentando (lo que puede incrementar su potencial impacto sobre la estabilidad financiera).

\section{Evolution of the links between banks and non-bank financial sectors in the Spanish financial system}

This paper describes the evolution of the interlinkages within the Spanish financial system (cross-sector dimension of systemic risk), distinguishing between direct and indirect links. The analysis concludes that the direct interconnections of the banking sector with the other sectors are low. Regarding indirect linkages, the relevance of non-banking sectors has been increasing (which may increase their potential impact on financial stability).

Palabras clave: estabilidad financiera, riesgo sistémico, solapamiento de carteras, correlación de carteras. Keywords: financial stability, systemic risk, portfolio overlap, portfolio correlation.

JEL: G18, G21, G22, G23, G28.

\footnotetext{
* Banco de España.

Contacto: patricia.stupariu@bde.es y jalonso.olmedo@bde.es

** Comisión Nacional del Mercado de Valores (CNMV).

Contacto: micambon@cnmv.es

Versión de octubre de 2020.

https://doi.org/10.32796/ice.2021.918.7157
} 


\section{Introducción}

El sistema financiero español está compuesto por varios tipos de intermediarios que, junto con las entidades bancarias, llevan a cabo operaciones esenciales para el funcionamiento de la economía como la concesión de financiación al sector público o privado, la gestión de patrimonios o el aseguramiento de actividades tanto comerciales como no lucrativas. El estudio de las interconexiones entre dichos intermediarios es relevante en el ámbito de la estabilidad financiera debido al riesgo de contagio entre los distintos sectores que estas pueden provocar en momentos de crisis, que constituye una parte de la dimensión transversal del riesgo sistémico (que interacciona con la temporal, aunque en este artículo se analizan por separado) ${ }^{1}$. Sin embargo, estos vínculos no deben interpretarse como una vulnerabilidad del sistema ya que su existencia también permite la diversificación de las fuentes de financiación en la economía.

En general, estos nexos se pueden clasificar en dos tipos. Por un lado, las interconexiones directas, que son las relaciones por las que dos entidades son contrapartes directas a través de instrumentos de deuda, acciones $u$ otras relaciones contractuales. Por otro lado, las interconexiones indirectas que son las que se establecen, por ejemplo, cuando las entidades mantienen exposiciones comunes a determinados sectores, mercados o instrumentos. Las vías a través de las que se pueden propagar los shocks en el sistema son múltiples y, según explican Freixas et al. (2015) «la distinción entre los distintos canales es más académica [y] suele ser difícil diferenciar un canal de otro, debido a que operan simultáneamente y se refuerzan recíprocamente». Estos autores mencionan siete tipos de canales que operan vía: $i$ ) la formación de expectativas, que generan pánicos bancarios en entidades solventes debido

\footnotetext{
1 Para más detalles sobre los canales de contagio y distintos métodos para la cuantificación de este riesgo, véase el capítulo 5 de Freixas et al. (2015), o Bricco y Xu (2019).
}

a problemas en otras entidades percibidas como similares; ii) riesgo de contraparte, que implica la potencial quiebra de varias entidades ante la quiebra de una contraparte relevante; iii) ausencia de liquidez, que puede llevar a que incluso entidades bien capitalizadas no puedan refinanciar sus deudas; iv) restricciones en la oferta de crédito ante la caída en el valor de ciertos activos que pueden reducir el capital bancario, por un lado, y el valor de las garantías de los acreditados, por otro (situación que puede surgir a raíz de un episodio de ventas forzadas); $v$ ) contagio de bancos a intermediarios financieros no bancarios (fondos de inversión, compañías de seguros, etc.); vi) publicación de información, disclosure, sobre el valor de mercado de los activos bancarios y del impacto en el capital que ciertas pérdidas pueden tener, con el subsiguiente aumento de los costes de financiación para las entidades afectadas y la disminución del margen financiero; o vii) contagio transfronterizo, cuando el contexto macrofinanciero internacional genera la fragilidad de varios sistemas financieros nacionales debido a la presencia en estos de bancos internacionales o a la existencia de una alta dependencia de la financiación del exterior.

En este trabajo se muestra la evolución en los últimos años de los principales tipos de interconexiones del sistema financiero español, centrándonos en interconexiones directas (tenencias cruzadas) e indirectas (similitud de las carteras de valores negociables de los principales sectores financieros). Para ello, se analizan datos sectoriales de las Cuentas Financieras e información granular sobre la composición de las carteras de valores negociables de bancos, compañías de seguros, fondos de inversión y fondos de pensiones.

Hasta ahora, la ausencia de bases de datos más granulares impedía desarrollar análisis muy detallados sobre estos aspectos, pero la disponibilidad de nuevas bases de datos (como la Securities Holdings Statistics by Sector, que utilizamos en este trabajo) permite explorar estos temas con mayor profundidad y llevar a cabo seguimientos periódicos de las interconexiones. La granularidad de estos datos permite identificar contrapartes, 


\section{FIGURA 1}

\section{SECTOR FINANCIERO EN ESPAÑA (2002 T3-2020 T1) - ACTIVOS FINANCIEROS} (En billones de euros)

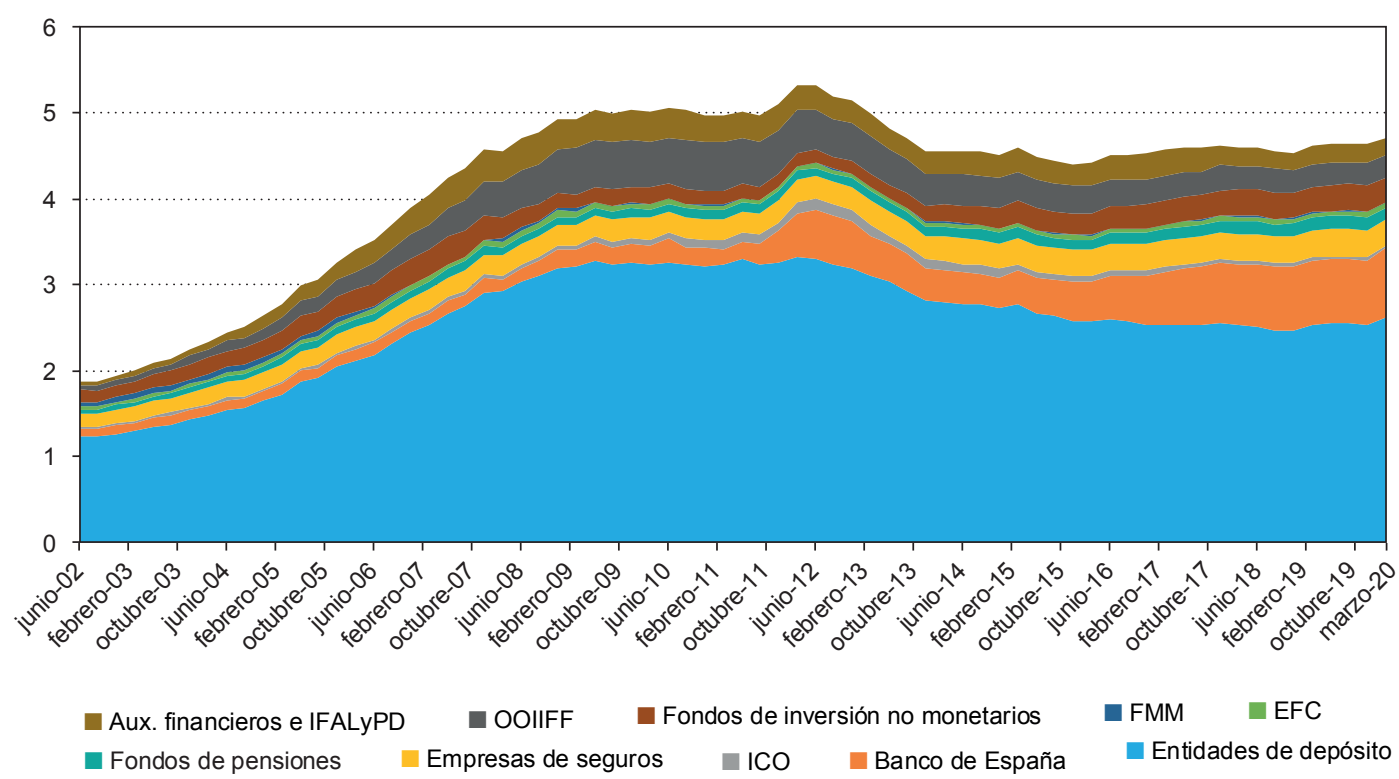

NOTA: ICO, Instituto de Crédito Oficial; FMM, Fondos de Mercado Monetario; OOIIFF, Otros Intermediarios Financieros; EFC, Establecimiento Financiero de Crédito; IFALyPD, Instituto Federal de Acceso a la Información y Protección de Datos. FUENTE: Banco de España. Cuentas Financieras Nacionales (los datos de las Cuentas Financieras son no consolidados y solamente cubren entidades domiciliadas en España).

sus nacionalidades y las características de los instrumentos que vinculan a unos agentes con otros.

Aunque en el sistema financiero español las entidades bancarias ocupan un papel central, existe una variedad de otros agentes que también llevan a cabo actividades clave $y$, en algunos casos, ofrecen financiación al resto de los agentes económicos, de manera similar a los bancos. Si atendemos al volumen de activos financieros que mantienen los distintos tipos de entidades, observamos que en los últimos años el peso del sector bancario se ha reducido. La Figura 1 muestra que mientras que dicho sector suponía el $65 \%$ del sistema financiero en 2002, a finales de 2019 representaba el 54,6 \%. Esto se debe a que, pese al crecimiento del sector hasta 2009, tras la crisis financiera global el total de activos mantenidos por los bancos se redujo. En todo este periodo, aparte del crecimiento del peso del Banco de España en el total del sector financiero (que pasó de ser del $5 \%$ en 2002 al $16 \%$ a finales de 2019), destaca el aumento de otros intermediarios financieros ${ }^{2}$ (que pasaron de suponer el $3 \%$ del sistema financiero en 2002 a representar el 5,5\% a finales de 2019). Pese a ello, la Figura 1 muestra que el aumento del peso de estos intermediarios fue interrumpido tras la crisis financiera y, desde entonces, se ha reducido.

\footnotetext{
2 La categoría de otros intermediarios financieros (OOIIFF - categoría S.125 de las Cuentas Financieras Nacionales) comprende a sociedades de valores, fondos de titulización de activos, sociedades de capital riesgo, fondos de activos bancarios, entidades de contrapartida central y sociedades de gestión de activos.
} 
También destaca la evolución de los fondos de inversión que tras su crecimiento a principios de este siglo (donde llegaron a suponer un $8 \%$ del sistema financiero) y su disminución tras la crisis, han mantenido una tendencia creciente que les ha permitido recuperar su relevancia. En concreto, a finales de 2019 estas entidades mantenían cerca del $7 \%$ de los activos financieros del sistema financiero frente a las cifras menores al 3 \% que llegaron a suponer en 2012.

Además, la Figura 1 permite observar la evolución del sistema financiero desde finales de 2019 hasta el primer trimestre de 2020, cuando el efecto de la pandemia y las medidas adoptadas para combatirla empezaron a hacerse sentir en la economía. El conjunto del sistema creció un $1,4 \%$ en este último trimestre (1,9\% interanual) debido al aumento de los activos mantenidos por los bancos $(3,6 \%)$ y por el banco central $(7,2 \%)$. El incremento de ambos compensó la caída experimentada por otros sectores como los fondos de inversión no monetarios (10,5\%) o los fondos de pensiones $(9,4 \%)$. Estas tendencias provocaron que el peso del sector bancario creciese hasta el 55,8 \%, lo que invertía la caída que venía experimentando desde 2011. Esto parece reflejar las diferencias del papel de las entidades bancarias en esta crisis, cuyo rol como intermediario se ha potenciado frente a otros sectores, respecto al que tuvieron en 2008. Esto se debe a que, mientras en 2008 la crisis se generó en el propio sector financiero, la actual crisis ocasionada por el coronavirus tiene su origen en una causa exógena al mismo.

Este contexto ha puesto de manifiesto la relevancia de las interconexiones dentro del sistema financiero, dado el riesgo de contagio entre diferentes agentes y la propagación de situaciones de estrés en el sistema. Los eventos e implicaciones provocados por la pandemia han comenzado a estudiarse $y$, organismos internacionales han identificado a las conexiones que facilitan la transmisión de riesgos como uno de los factores que merecen especial atención. Así, por ejemplo, el Consejo de Estabilidad Financiera (FSB, por sus siglas en inglés) inició en 2020 un proyecto para cartografiar las interconexiones dentro del sistema financiero que permitirá identificar los nexos entre las distintas partes del sistema. Este trabajo continúa la labor que algunos de estos organismos habían emprendido tras la crisis de 2008 para mejorar el seguimiento, el análisis y el marco regulatorio asociados. Por ejemplo, desde 2011, el FSB ha publicado su informe anual de seguimiento del sector no bancario, en el que una sección hace siempre una revisión de las interconexiones dentro del sector financiero como indicadores relevantes del potencial riesgo de contagio. A escala europea, la Junta Europea de Riesgo Sistémico (ESRB, por sus siglas en inglés) publica, desde 2016, un informe anual de seguimiento del sector no bancario que también centra parte de sus esfuerzos en analizar las interconexiones y el riesgo de contagio dentro del sector financiero. Finalmente, el Banco Central Europeo (BCE) ha incorporado también análisis sobre el sector financiero no bancario en su Informe de Estabilidad Financiera ${ }^{3}$.

Las interconexiones entre los distintos sectores financieros son un desarrollo natural en cualquier sistema financiero maduro y pueden contribuir a la diversificación de las fuentes de financiación y del riesgo entre agentes. Sin embargo, como ocurrió en la crisis financiera global, los desequilibrios o shocks generados en un sector (o grupo concreto de entidades de un sector) pueden trasladarse al resto del sistema financiero. Este riesgo de contagio puede ser más pronunciado cuanto más largas y complejas sean las cadenas de intermediación de crédito, dada la menor capacidad de establecer cortafuegos a tiempo y al desconocimiento de sus características.

Acontinuación, se describen en primer lugar las interconexiones directas en el sistema financiero español

\footnotetext{
3 Además, algunos de los apartados de la reforma regulatoria de los últimos años han abordado los riesgos generados a través de dichas interconexiones: desde las medidas para fortalecer los marcos para los fondos del mercado monetario o para las titulizaciones, hasta las desarrolladas para mitigar riesgos en los mercados de préstamo de valores, o los apartados de Basilea III que limitan determinados tipos de exposiciones.
} 
y, en segundo lugar, las indirectas. Finalmente, se presentan las principales conclusiones del trabajo.

\section{Interconexiones directas}

A la hora de profundizar en el análisis, conviene partir de la tipología de interconexiones utilizada habitualmente en los trabajos sobre este tema. Los estudios previos sobre interconexiones tienden a centrarse en las interconexiones directas, principalmente por la mayor disponibilidad de información sobre las mismas. Estas son relaciones por las que dos entidades son contrapartes directas a través de instrumentos de deuda, de patrimonio $u$ otras relaciones contractuales en los mercados financieros. En general, los análisis de estas conexiones se centran en las tenencias cruzadas: emisiones de una entidad financiera en manos de otra entidad financiera, perteneciente al mismo o a otro sector financiero; o exposiciones crediticias directas dentro del mismo sector o entre distintos sectores. Ese análisis de tenencias cruzadas es el que desarrollamos en este apartado, sin profundizar en otro tipo de conexiones directas como las generadas, por ejemplo, por los ingresos que suponen para los bancos actividades como la gestión de fondos de inversión o la oferta de productos de seguros (es decir, interconexiones vía ingresos y gastos en la cuenta de resultados).

Las Cuentas Financieras Nacionales permiten hacer seguimiento de estas conexiones y su evolución. Dichos datos reflejan que la importancia que cada sector tiene en el sistema financiero debido a su tamaño, se refleja también en su nivel de interconexiones con los otros sectores. Es decir, los sectores más grandes suelen tener una posición más relevante en un mapa de las interconexiones. De esta manera, el sector bancario, que en España predomina respecto al resto de tipos de entidades, es el que cuenta con un volumen de interconexiones directas más significativo. Dado el rol central de este sector, a continuación se analizan sus nexos con otras áreas del sistema y su evolución temporal.
Pese a ello, las interconexiones directas de los bancos con cada uno de los otros sectores representan un porcentaje relativamente reducido del activo financiero bancario, que en ningún momento supera el $5 \%$ de dichos activos financieros. Las principales interconexiones se dan entre bancos y Otras Instituciones Financieras (OIF), siendo los activos de los bancos con las OIF equivalentes a alrededor del $4 \%$ del activo financiero total de los bancos y los pasivos con estas instituciones, cercanos al $3 \%$ del activo financiero bancario. Respecto a su evolución, España no sigue la tendencia global descrita en los informes del FSB caracterizada por un crecimiento de las interconexiones entre bancos y OIF en los años previos a la crisis y una caída a partir de 2009. Así, en España el nivel de interconexiones entre bancos y OIF se mantiene relativamente estable: en torno al $4 \%$ y con una tendencia decreciente desde la crisis financiera (para los pasivos frente a OIF), y al $3 \%$ y $4 \%$ (para los activos) de los activos financieros de las entidades bancarias.

Durante los últimos años se ha registrado cierta estabilidad de las series con una tendencia a la baja de los pasivos de los bancos frente a OIF, aseguradoras y fondos de pensiones. Este resultado indica que la dependencia de las entidades bancarias respecto a la financiación recibida de estos otros sectores se está reduciendo. Si atendemos a la evolución en el primer trimestre de 2020, tampoco se aprecian cambios significativos. Aunque se han observado ligeras evoluciones en los volúmenes, en términos del porcentaje de activos financieros de los bancos estas cifras apenas han variado.

Pese a que estas cifras indican que las conexiones dentro del sistema financiero español están contenidas, hay que tener en cuenta que esta descripción se basa en cifras agregadas. Para lograr una evaluación más precisa de los posibles canales de transmisión y los riesgos asociados hay que tener en cuenta elementos adicionales. Por ejemplo, en primer lugar, es clave analizar la concentración de estos nexos, ya que podrían generar vulnerabilidades para entidades concretas. En segundo lugar, el vencimiento a más corto o largo 
FIGURA 2

INTERCONEXIONES DIRECTAS DE BANCOS CON OTROS SECTORES POR INSTRUMENTO (MARZO 2020)
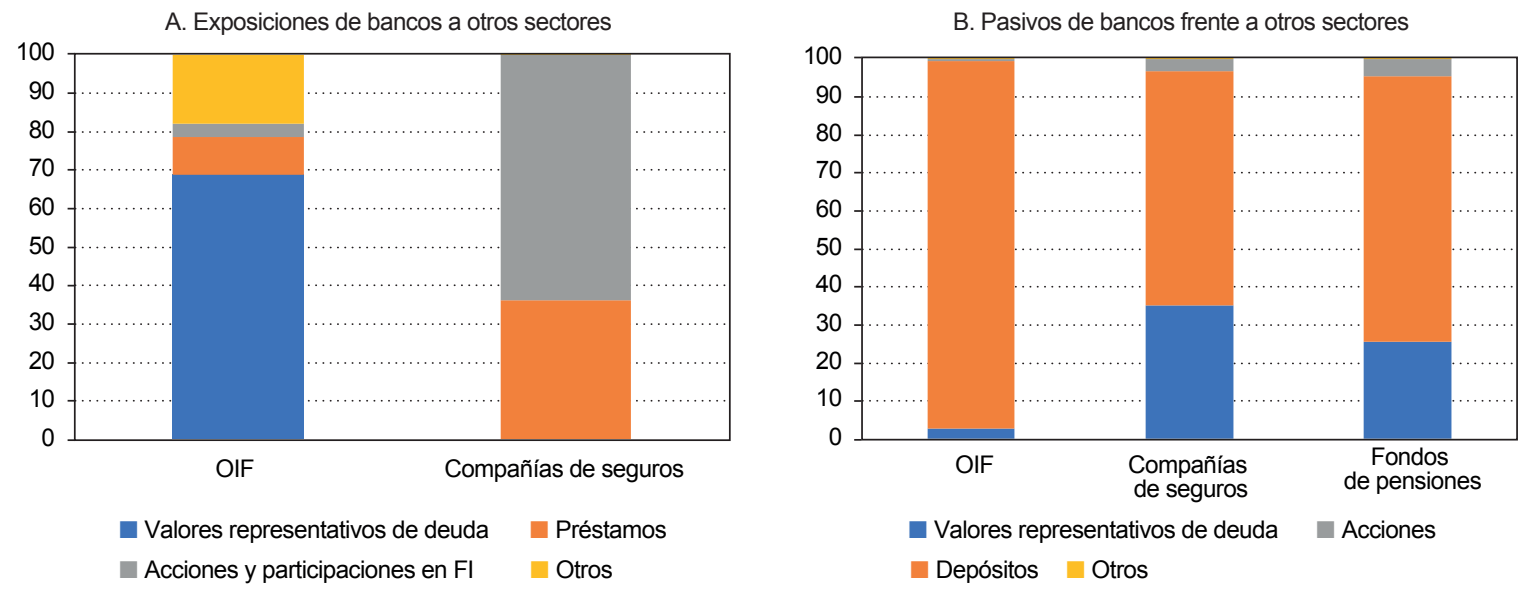

NOTA: Las siglas OIF se refieren a Otras Instituciones Financieras, categoría que comprende varios sectores de las cuentas financieras: otros intermediarios financieros (tales como sociedades de valores, fondos de titulización de activos, sociedades de capital riesgo, fondos de activos bancarios, entidades de contrapartida central o sociedades de gestión de activos), establecimientos financieros de crédito y fondos de inversión (monetarios y no monetarios).

FUENTE: Banco de España. Cuentas Financieras Nacionales.

plazo de los instrumentos a través de los que se producen estas interconexiones también influirá en los riesgos asociados. Finalmente, la existencia de conexiones de las entidades domiciliadas en España con sectores extranjeros (para las que las Cuentas Financieras no proporcionan datos desagregados por la tipología del sector no residente) supone otro aspecto a analizar.

Aparte de los volúmenes, la tipología de estas conexiones también proporciona información útil para comprender su comportamiento (Figura 2). Si atendemos a las exposiciones de los bancos a otros sectores se observa que, en marzo de 2020, la mayor parte de las exposiciones del sector a OIF es a través de instrumentos de deuda (69\%). El peso de los préstamos (10\%) a las entidades de este sector es menor que el que tiene en las exposiciones a compañías de seguros (36 \%). La mayor parte de las exposiciones al sector asegurador es a través de renta variable (64\%).
Si se atiende a los pasivos, destaca la relevancia de los depósitos. Sin embargo, mientras que la práctica totalidad de los pasivos de bancos frente a OIF son depósitos, el peso de los valores de deuda es más significativo para compañías de seguros (35 \%) y fondos de pensiones (26\%).

En el comienzo de 2020, tanto los pasivos como los activos de bancos frente a OIF se redujeron, aunque la caída de los pasivos fue más pronunciada (superior al $7 \%$, lo que refleja disminuciones en todas las categorías de instrumentos). Los depósitos de compañías de seguros y fondos de pensiones se mantuvieron más estables, mientras que los préstamos a estas entidades y las tenencias de deuda bancaria por parte de las mismas sí que se redujeron de forma más significativa durante el primer trimestre de 2020 (con caídas en torno al 10 \% para ambos instrumentos). 
Otra área de interés es el de las conexiones que tiene el sistema financiero doméstico con las entidades extranjeras, tanto bancos, como no bancos. Según muestran varios análisis recientes, a nivel global, los vínculos transfronterizos entre el sector bancario y otros sectores financieros han estado aumentando en los últimos años, sobre todo en las economías más desarrolladas financieramente (Aldasoro et al., 2020).

\section{Interconexiones indirectas}

Además de las conexiones directas descritas en el apartado anterior, pueden existir distintos tipos de interconexiones indirectas entre los sectores financieros (o entre entidades de un mismo sector). Estas conexiones son las que se establecen cuando las entidades financieras mantienen exposiciones comunes a determinados sectores, mercados o instrumentos; participan en cadenas de colateral; pertenecen a los mismos grupos corporativos; o están expuestas al riesgo reputacional fruto del apoyo financiero a filiales o a otras entidades dependientes, más allá de las relaciones contractuales. En momentos de crisis, estos nexos pueden provocar que entidades que inicialmente no presentaban problemas, se vean afectadas debido a este segundo tipo de vínculos. En este apartado, se tratará con más detalle el solapamiento y la correlación de carteras, incluyendo un recuadro que analiza la correlación entre distintos tipos de activos, basado en la información de mercado. A continuación, se analiza la composición de la cartera de valores negociables de los bancos, compañías de seguros, fondos de inversión y fondos de pensiones; y el grado de similitud de las mismas en cuanto a tipo de instrumentos y emisores.

\section{Solapamiento de carteras}

Los distintos sectores financieros mantienen títulos similares en sus carteras (emitidos por sectores financieros o no financieros) y son estas tenencias comunes las que dan lugar a lo que denominamos solapamiento de carteras, que puede llegar a convertirse en un mecanismo de contagio, que actúa por múltiples canales. Por ejemplo, ante un shock en el sector de los fondos de inversión, estos podrían verse en la necesidad de vender activos, en situaciones de reducida liquidez de los mercados, que están también presentes en las carteras de bancos o aseguradoras, pudiendo generar caídas de los precios de estos activos y pérdidas de valoración para estos otros sectores, lo que puede tener implicaciones para la estabilidad financiera.

Otro canal es el impacto de la caída de los precios en el valor de las garantías depositadas en operaciones financieras cuando las mismas consisten en los activos objeto de las ventas forzadas $u$ otros impactados por dichas ventas. A los depositantes de estas garantías se les exigirá aportar garantías adicionales que pueden no estar inmediatamente disponibles. De la misma forma, que los inversores pasen a considerar algunos activos como tóxicos/de baja calidad también puede tener un impacto sobre los sectores que mantienen dichas exposiciones o que se financian a través de dichos títulos (incluso aunque existan diferencias entre clases de activos si no existe información que permita discriminar).

La Figura 3 muestra la disminución del tamaño de la cartera del sector bancario de los últimos años, mientras que las carteras de compañías de seguros y fondos de inversión han aumentado y la de los fondos de pensiones se ha mantenido relativamente estable 4 . La cartera de valores negociables representa un $23 \%$ del total del activo financiero de los bancos, mientras que para las compañías de seguros (CS) representa un $82 \%$, para los fondos de inversión (FI) un $80 \%$ y para los fondos de pensiones (FP) un $78 \%$.

La Figura 4 muestra la evolución en los últimos siete años del porcentaje de tenencias comunes de

\footnotetext{
4 El contenido de este apartado ha sido elaborado en base a la información procedente de Securities Holdings Statistics by Sector (SHSS), una base de datos gestionada por el Banco Central Europeo, que contiene información sobre la composición de las carteras de los distintos sectores financieros de la zona euro.
} 
FIGURA 3

\section{CARTERA DE VALORES DE LOS SECTORES FINANCIEROS DOMÉSTICOS}
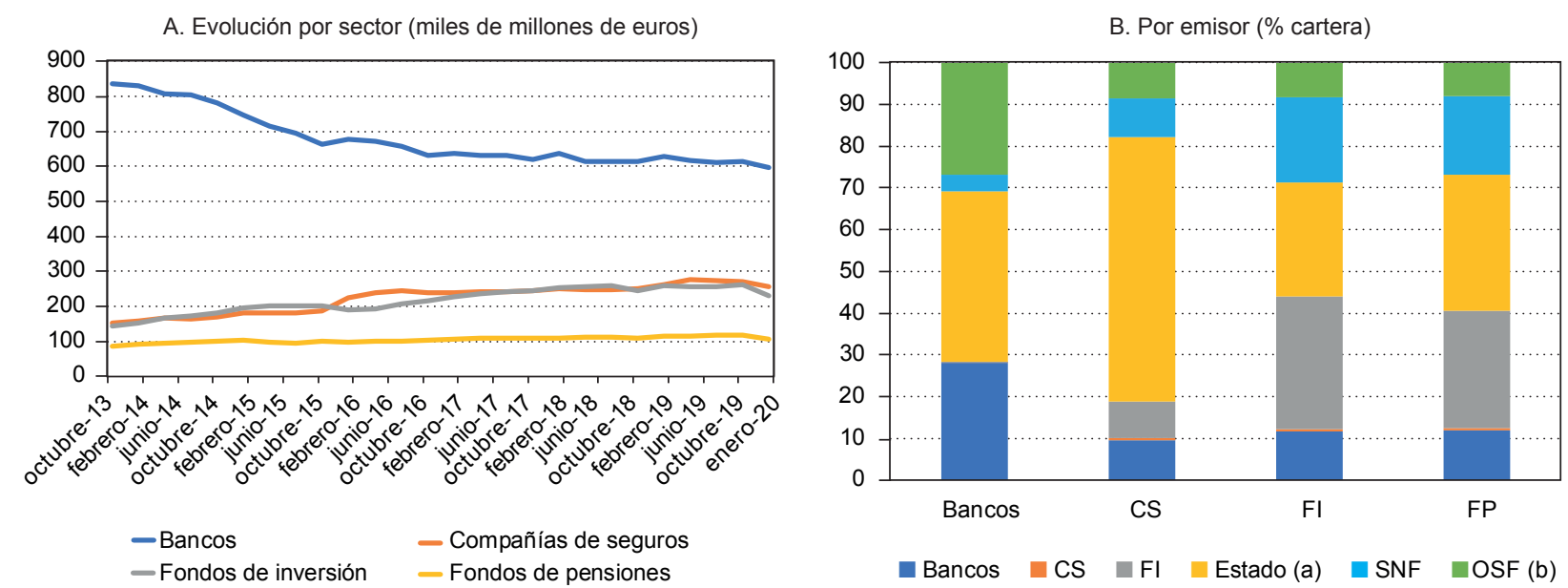

NOTA: El valor de la cartera de valores negociables de cada sector se calcula a precios de mercado (o valor razonable). Cabe resaltar que el análisis se centra en las carteras de valores negociables y no en el total de activos. Esto es relevante para algunos sectores como el bancario ya que dicha cartera tiene un peso limitado dentro de su total de activos. En La Figura 3B, los acrónimos CS se refieren a compañías de seguros; FI, fondos de inversión; FP, fondos de pensiones; SNF sociedades no financieras.

a) La categoría «Estado» incluye emisores soberanos y Administraciones locales.

b) La categoría "OSF» se refiere a Otros sectores financieros: auxiliares financieros, instituciones financieras con actividad limitada y prestamistas de dinero y otros intermediarios financieros.

FUENTE: Securities Holdings Statistics by Sector (SHSS).

títulos negociables (excluyendo préstamos) para cuatro sectores del sistema financiero español (bancos, compañías de seguros, fondos de inversión y fondos de pensiones). Dichos porcentajes representan el peso que constituyen las tenencias comunes entre cada par de sectores en la cartera de cada sector. Se observa una tendencia decreciente de esta medida de similitud de carteras a lo largo del tiempo, excepto para las compañías de seguros, cuyas tenencias comunes con el resto de sectores se ha mantenido a un nivel más estable. Lo que más disminuye es el solapamiento del sector bancario con el resto de sectores, con la excepción de lo que supone el solapamiento con los bancos para las compañías de seguros.

Para algunos de los sectores, gran parte del solapamiento se debe a la tenencia de bonos soberanos, sobre todo emitidos por gobiernos de la eurozona $\mathrm{y}$, en particular, por instituciones públicas españolas (principalmente bonos soberanos $y$, en menor medida, bonos emitidos por Administraciones locales). Esto es coherente con la distribución de las carteras de cada sector por emisor de los títulos que mantienen. Como muestra la Figura 3B, los fondos de inversión y los fondos de pensiones mantienen carteras más diversificadas en cuanto a sectores (aunque no se incluye en la Figura 3B, nuestros cálculos también muestran carteras más diversificadas en cuanto a geografías).

El solapamiento a nivel de títulos individuales muestra una imagen parcial de las interconexiones indirectas debido a que sectores distintos pueden invertir en títulos de distinta naturaleza emitidos por el mismo emisor (bonos frente a acciones, bonos a 


\section{FIGURA 4}

\section{EVOLUCIÓN DE LAS TENENCIAS COMUNES DE TÍTULOS POR SECTOR (En \% respecto a la cartera de cada sector)}
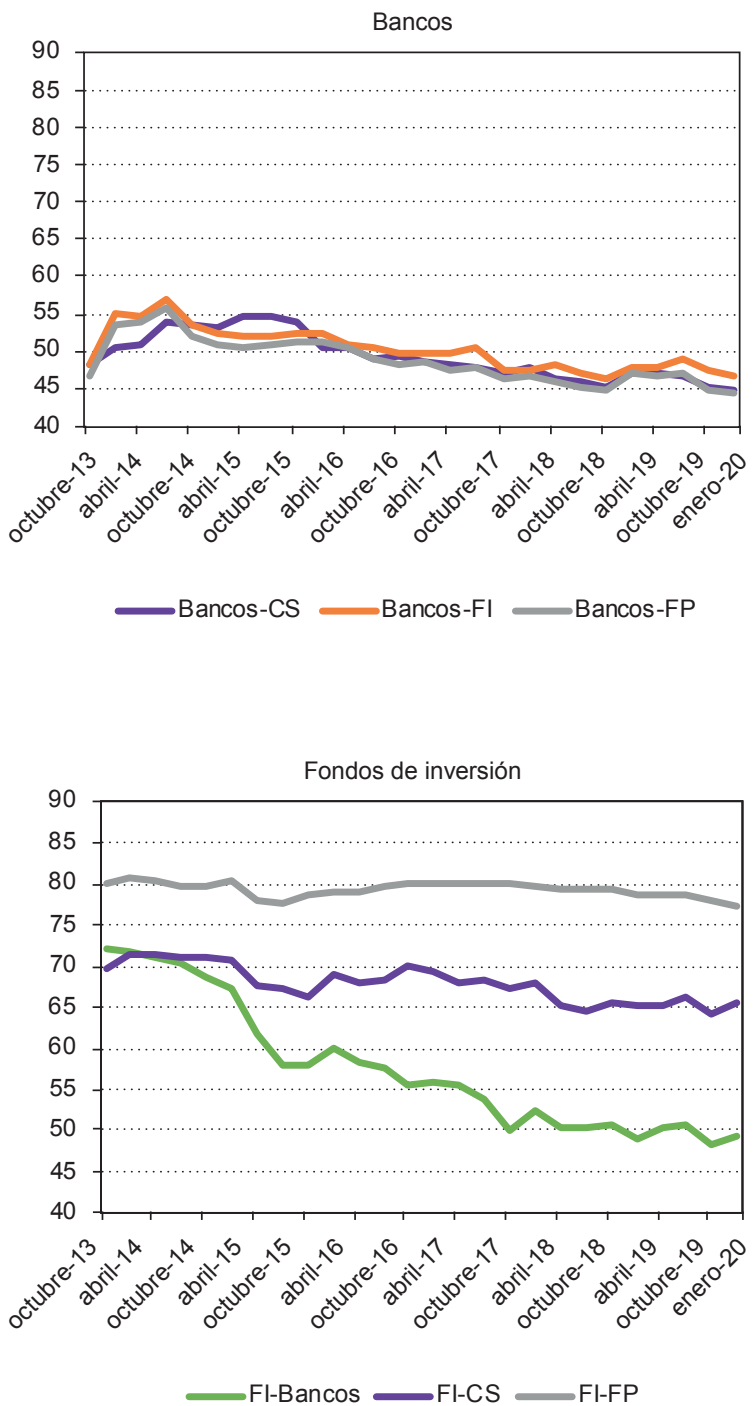
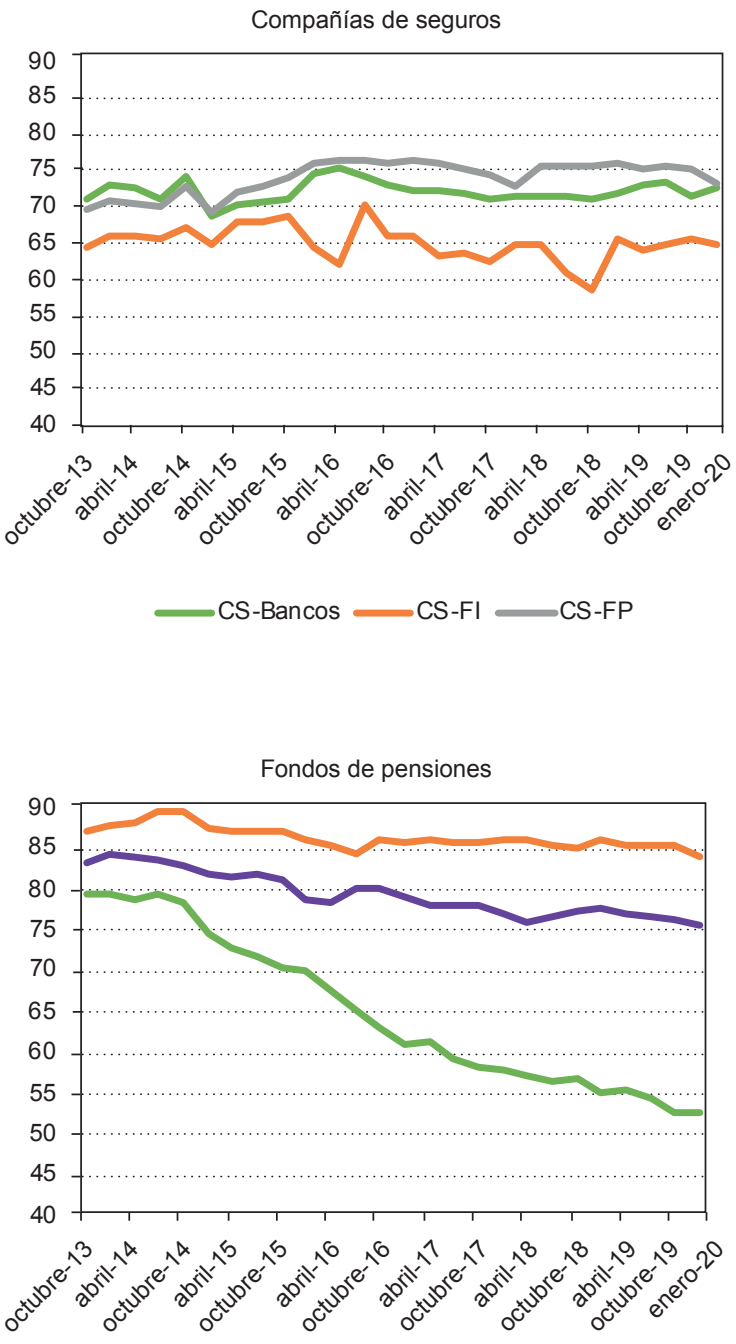

FP-Bancos FP-CS FP-FI

NOTA: Para cada sector, se muestra el volumen de tenencias en su cartera de valores negociables que también mantiene cada uno de los otros tres sectores (el análisis recoge el solapamiento a nivel de títulos individuales). Por ejemplo, si un banco y un fondo de inversión mantienen en la cartera el mismo título de deuda emitido por una sociedad no financiera (identificado a través del código único asignado a cada emisión), los importes que mantienen el banco y el fondo de inversión de dicha emisión —en general, no mantienen el mismo volumen de cada título común-computan para la medida de solapamiento, que se expresa como porcentaje de la cartera total de cada sector. En la última fecha disponible, finales de T1 2020, de las tenencias comunes entre bancos y fondos de inversión, los bancos mantienen en sus carteras el equivalente al $46 \%$ del total de su cartera. Por su parte, los fondos de inversión mantienen el equivalente al $50 \%$. Se toma el valor de mercado de las tenencias que reportan las entidades (o el valor razonable, en su caso).

FUENTE: Securities Holdings Statistics by Sector (SHSS). 
FIGURA 5

COEFICIENTES DE CORRELACIÓN ENTRE CARTERAS DE SECTORES (2013 T4-2020 T1)

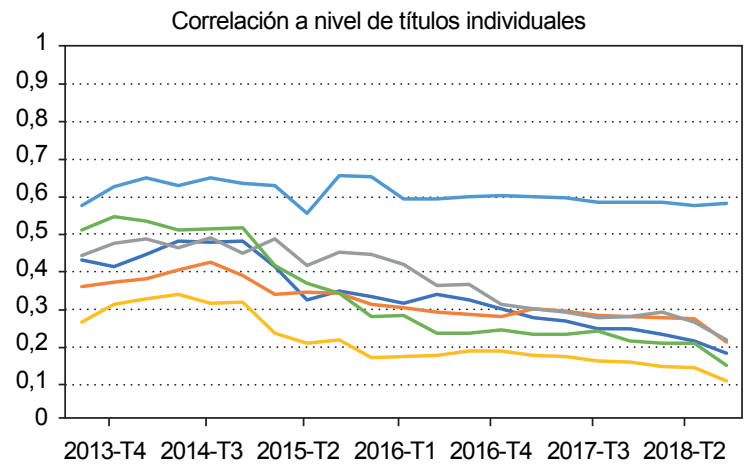

-Bancos - FI -Bancos - CS -Bancos - FP

$-\mathrm{FI}-\mathrm{CS}-\mathrm{FI}-\mathrm{FP}-\mathrm{CS}-\mathrm{FP}$

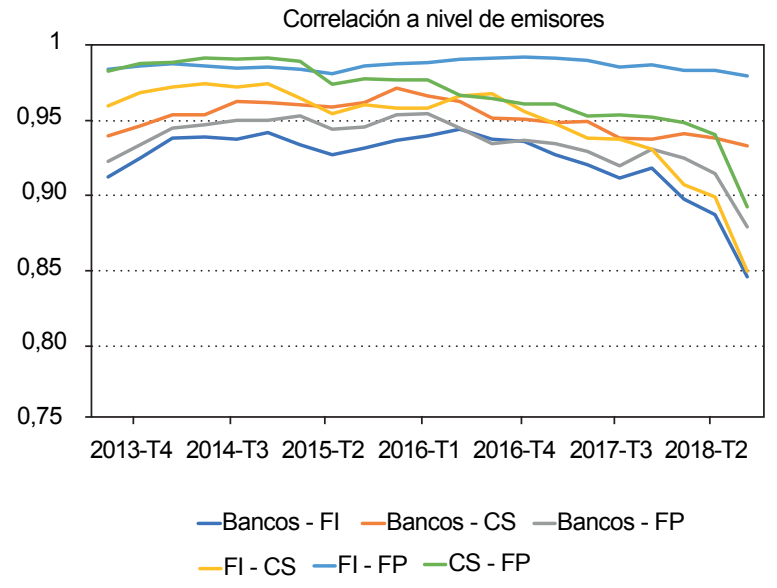

FUENTE: Securities Holdings Statistics by Sector (SHSS). corto plazo o bonos a largo plazo, etc.). Por tanto, cuando el solapamiento se mide a nivel de emisor -sumando el valor del total de las tenencias comunes asociadas a un mismo emisor- dicho solapamiento de carteras aumenta. Por ejemplo, el solapamiento entre bancos y fondos de inversión calculado a nivel de emisor, aumenta hasta el $73 \%$ de su cartera para bancos y $65 \%$ para fondos de inversión. Como en el caso del solapamiento expresado a nivel de títulos individuales, una parte relevante de esta medida refleja tenencias de bonos soberanos emitidos por el Estado español. En función del análisis que se quiera realizar, ambas perspectivas pueden ser de utilidad.

\section{Correlación de carteras}

Una medida adicional para aproximar la similitud entre las carteras es el coeficiente de correlación de las tenencias de cada par de sectores. De esta manera, en cada fecha podemos estimar la medida en la que las carteras están distribuidas de forma similar entre los distintos títulos que las componen ${ }^{5}$. Esta medida no depende del tamaño de las carteras $y$, por lo tanto, no está afectada por las diferencias en el volumen total de tenencias de cada sector.

Una correlación positiva entre dos sectores indicaría, por ejemplo, que las tenencias cuyo volumen es superior a la media del total de la cartera en uno de los sectores se encuentran, en general, con un valor superior a la media también en la cartera del otro sector. Por el contrario, una correlación negativa indicaría que las tenencias cuyo volumen es inferior a la media de la cartera en uno de los sectores tienen un valor superior a la media en el otro sector.

\footnotetext{
5 Se calcula el coeficiente de correlación de Pearson entre cada par de sectores en cada fecha, utilizando el valor de mercado de las tenencias de cada título individual (expresado en euros) que se encuentra en la cartera de los sectores financieros. El coeficiente de correlación se define como la ratio entre la covarianza de las tenencias de cada par de sectores y el producto de las varianzas de dichas tenencias en cada fecha.
} 


\section{TABLA 1 \\ UN ANÁLISIS DE LAS INTERCONEXIONES BASADO EN LA INFORMACIÓN QUE PROPORCIONAN LOS MERCADOS FINANCIEROS}

Los análisis relacionados con la medición de las interconexiones indirectas pueden ser de diversa naturaleza. Además de analizar la existencia de carteras de valores con posiciones comunes en determinados instrumentos financieros o la correlación entre las carteras de los diferentes sectores, también resulta interesante estudiar la dinámica de los precios de diferentes tipos de activos en los mercados financieros a través de su correlación ${ }^{1}$. Este análisis permite conocer hasta qué punto los precios de los activos financieros se mueven en la misma dirección, de manera que un valor alto en el nivel del indicador estimado incitaría a pensar que las ventajas de la diversificación de carteras para los inversores se reducen pues es más difícil reducir la exposición a fuentes de riesgo sistemático.

Además, en un entorno de correlación alta es concebible pensar que, ante un shock negativo en alguno de los activos o de un conjunto de ellos, se pueden producir descensos más o menos generalizados en el precio del resto de instrumentos. Este análisis es especialmente relevante en momentos de turbulencias o de estrés en los mercados en los que el aumento de incertidumbre de los agentes suele dar lugar a ventas generalizadas de activos, con independencia de las vulnerabilidades que se identifiquen sobre los mismos, y puede tener, en último caso, consecuencias importantes desde el punto de vista de la estabilidad financiera.

En la Figura 6 se presenta el resultado de este análisis de correlaciones realizado con datos de precios diarios en ventanas de tres meses. Para ello se calculan todos los pares de correlaciones posibles entre seis tipos de activos financieros: deuda soberana, renta fija privada de entidades financieras, renta fija de entidades no financieras y valores del lbex 35 de compañías financieras, de utilities, y del resto de sectores y se presenta la mediana, así como la información de otros percentiles.

Como se observa en la Figura 6, se pueden identificar, a grandes rasgos, tres periodos diferentes en la evolución de dichas correlaciones. En los primeros años del análisis, entre 2010 y 2016, la mediana de las correlaciones entre los activos de renta variable y renta fija se mantuvo, con ciertas oscilaciones, en valores que oscilaron entre 0,4 y 0,6. En el último año de este periodo, 2016, la correlación entre los activos aumentó coincidiendo con el anuncio del Reino Unido de su decisión de dejar de formar parte de la Unión Europea. Esta decisión originó un periodo de turbulencias en los mercados financieros que se caracterizó por las caídas generalizadas en los precios de los activos. La correlación relativamente alta de estos años, en términos generales, se debe a la conjunción de caídas en los precios de los activos de renta fija y variable en el periodo 2010-2012 en el contexto de la crisis de la deuda soberana y de aumentos con posterioridad a la misma. Cabe señalar que el rendimiento de la deuda pública española a los diez años disminuyó de forma casi continua desde el 7,6\% a mediados de 2012, hasta el 1,2\% a principios de 2015.

En el segundo periodo, desde finales de 2016 hasta el inicio de la crisis derivada del coronavirus, se observó un descenso, con altibajos, de la mediana de las correlaciones hasta valores cercanos a cero al final de dicho periodo, así como un fuerte aumento de la diferencia entre la correlación mínima y máxima. En este periodo de tiempo, los tipos de interés mostraron mucha más estabilidad (en el caso de la deuda pública a diez años los rendimientos oscilaron entre el $1 \%$ y el $2 \%$ la mayor parte del periodo, acercándose a cero en 2019) mientras que en las bolsas se observan revalorizaciones al principio y descensos después. A finales de este periodo se identifican claramente correlaciones negativas de cierta intensidad entre los precios de los activos de deuda pública y los de otros activos, tanto de renta fija privada como de renta variable (especialmente financiera).

El tercer periodo, que comienza con el inicio de la crisis en marzo de 2020, se caracteriza por un aumento sustancial en el nivel de las correlaciones hasta máximos algo inferiores a 0,80 (en mediana), así como una disminución de la diferencia entre la correlación mínima $(0,41)$ y la máxima $(0,89)$. El fuerte aumento de la correlación en los precios de los activos financieros españoles en los primeros momentos de la crisis estuvo asociado a la caída tanto de las cotizaciones bursátiles como de los precios de los activos de deuda, con repuntes importantes en las primas de riesgo de algunos segmentos. Esta correlación ha tendido a reducirse pues los activos de renta variable han seguido una tendencia irregular, a la baja en algunos sectores importantes como el financiero, mientras que la mayoría de los activos de deuda han mostrado aumentos en sus precios como consecuencia de las decisiones adoptadas por el BCE y materializadas, fundamentalmente, en compras significativas de distintos activos de renta fija.

1 Correlación no implica causalidad y, por tanto, esto no necesariamente implica que, ante un shock idiosincrático en un activo, todo el resto de activos se vayan a ver afectados con la intensidad que muestran esas correlaciones.

Adicionalmente, cuanto menor sea la dispersión del valor de las tenencias de cada cartera alrededor del valor medio de las mismas, más alta será la correlación entre las carteras.

El panel izquierdo de la Figura 5 refleja la evolución de los coeficientes de correlación calculados a nivel de títulos individuales (el panel derecho, a nivel de emisor), desde el cuarto trimestre de 2013 hasta principios de 2020. Se observa que la mayor correlación a lo largo de todo el periodo analizado se da entre las carteras de fondos de inversión y fondos de pensiones. Además, mientras que para el resto de pares esa 
TABLA 1 (Continuación)

\section{UN ANÁLISIS DE LAS INTERCONEXIONES BASADO EN LA INFORMACIÓN QUE PROPORCIONAN LOS MERCADOS FINANCIEROS}

\section{FIGURA 6}

\section{CORRELACIONES ENTRE CLASES DE ACTIVOS (OCT 2010 - OCT 2020)}

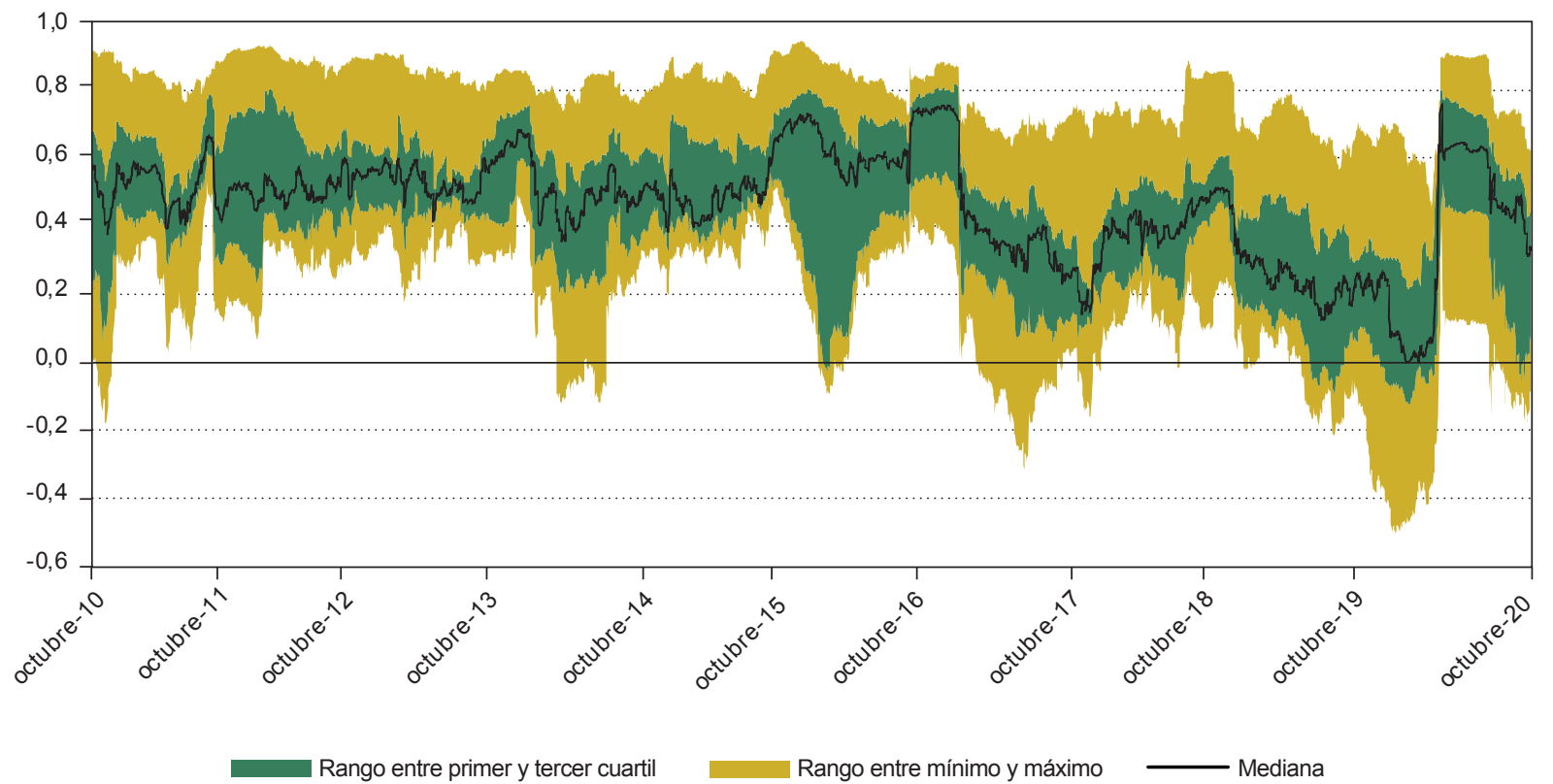

FUENTE: Elaboración propia a partir de datos de la CNMV y Thomson Datastream.

correlación ha seguido una tendencia decreciente, la existente entre las carteras de fondos de inversión y fondos de pensiones ha mantenido una evolución más estable en torno al $60 \%$.

La correlación calculada a nivel de emisores (panel derecho de la Figura 5) muestra también una tendencia decreciente a lo largo del tiempo, pero desde valores considerablemente más elevados ${ }^{6}$. Como en el caso de las medidas de similitud, el aumento de la

6 Igual que en el caso de la medida de solapamiento, el cálculo de la correlación a nivel de emisores (agrupando todos los títulos emitidos por un único emisor) redunda en unos mayores coeficientes de correlación entre las carteras de los distintos sectores. correlación calculada a nivel de emisor se debe principalmente a que se agrupan todas las tenencias comunes asociadas a un mismo emisor que pueden ser de distinta naturaleza (bonos, acciones o participaciones en fondos de inversión) o tener distintas características (por ejemplo, distintos vencimientos).

\section{Conclusiones}

En los últimos años, el estudio de los distintos componentes del sistema financiero ha sido el objeto de numerosos trabajos. El análisis de las interconexiones entre dichos componentes, llevado a cabo en los 
apartados anteriores, facilita la comprensión de la arquitectura del sistema financiero doméstico. Este trabajo muestra que las interconexiones directas del sector bancario con los otros sectores financieros se han mantenido relativamente estables en los últimos años, y a un nivel reducido cuando se comparan con el tamaño del activo bancario. A su vez, las interconexiones indirectas de los bancos a través de tenencias comunes con los otros sectores se han ido reduciendo (aunque, en relación al total de la cartera de valores negociables, siguen en niveles significativos en algunos casos, sobre todo cuando se miden a nivel de emisores). Los sectores no bancarios están más interconectados desde el punto de vista de la similitud de sus carteras y, aunque su peso en nuestro sistema financiero es menor que el del sector bancario, su relevancia ha venido aumentando. Esta tendencia puede provocar también un mayor potencial por su parte para generar problemas para la estabilidad financiera.

Sin embargo, los canales de contagio propiciados por las interconexiones en situaciones de crisis pueden ser muy variados e ir más allá de los expuestos en los apartados anteriores. Por ejemplo, en un momento de tensión en los mercados y caída de los precios de los activos financieros, el aumento de las exigencias de garantías, margin calls, por cambios en el valor de los derivados compensados en cámaras de compensación puede generar reacciones en cadena: los agentes que tuvieran una débil posición de liquidez, ante un aumento de los requerimientos de garantías podrían desinvertir o dejar de financiar a otros sectores abruptamente. Si estas dinámicas llevaran, por ejemplo, a retirar dinero de los fondos de inversión, estos podrían verse en la necesidad de realizar ventas forzadas si sus propias posiciones de liquidez fueran insuficientes para hacer frente a las retiradas. Estos problemas pueden ser agravados por la caída en el valor de las garantías y el aumento de los haircuts. Otras maneras a través de las cuales se puede propagar el contagio son las situaciones en las que las dudas sobre una entidad concreta, en base a unas similitudes superficiales o por pertenencia a un sector determinado, se extienden a todo un sector, independientemente de los vínculos efectivos que pueden existir dentro del mismo sector.

Los acontecimientos de los últimos meses a raíz de las turbulencias generadas por la crisis sanitaria han puesto de manifiesto, entre otros, algunos de los canales anteriormente mencionados y también la necesidad de llevar a cabo análisis periódicos que permitan identificar las vulnerabilidades más relevantes que podrían ser amplificadas a través de las interconexiones dentro del sistema financiero. En este contexto, el mapeo y estudio de las interconexiones como canales de transmisión es necesario. Sin embargo, dentro de una misma estructura de interconexiones, el comportamiento de los agentes durante una crisis estará condicionado también por otros factores, como los apoyos públicos, la política monetaria (incluyendo la no convencional) $u$ otros acontecimientos en el contexto internacional.

Cuantificar la magnitud de la amplificación o mitigación de shocks dentro del sistema financiero debido al solapamiento de carteras no es inmediato. En general, los análisis a nivel sectorial podrían sobreestimar o subestimar el grado en el que las ventas de un sector podrían generar ventas en otros sectores. Por ejemplo, si las tenencias estuvieran repartidas entre un gran número de entidades de un sector, el impacto de unas ventas forzadas, fire sales, en otro sector podría no ser tan elevada. Sin embargo, si las tenencias comunes están más concentradas, algunas entidades podrían sufrir pérdidas relevantes $y$, en un escenario estresado, generar contagio a otras entidades del mismo sector por vías como las retiradas de depósitos o la reticencia de los inversores de refinanciar al sector en cuestión. Para analizar más en profundidad el impacto de unas ventas forzadas, a menudo, se hace énfasis en las calificaciones de las tenencias comunes, siendo los activos más próximos al límite inferior del grado de inversión los que podrían generar mayores niveles de ventas si su calificación bajara por debajo de este umbral. Pero 
ante una crisis severa y prolongada, también se pueden registrar rebajas de calificación de títulos o emisores con calificaciones superiores. Por último, el impacto inmediato de un deterioro en los precios de mercado o en la calidad crediticia de los emisores dependerá también del método de contabilización de los activos (a precios de mercado o coste amortizado).

Para poder aprovechar todas las posibilidades de la creciente disponibilidad de información es necesario combinar distintas bases de datos y, debido a la globalización del sistema financiero, también se requiere una creciente cooperación e intercambio de información entre supervisores que permita identificar focos de riesgos y canales de transmisión de los mismos. La existencia de bases de datos como SHSS, facilita también análisis de escenarios y de impacto de eventos recurrentes en las crisis, como las rebajas de calificación de los emisores más vulnerables, las ventas forzadas y las pérdidas que estos pueden generar ${ }^{7}$.

Pese a esta creciente cantidad de datos, aún existen lagunas de información que deben ser abordadas. Por ejemplo, los datos utilizados no ofrecen información sobre las carteras al máximo nivel de consolidación, estando disponibles solo datos de tenencias de entidades residentes, lo que impide excluir interconexiones intragrupo.

En este contexto, cabe resaltar también la importancia de las conexiones transfronterizas. El análisis descrito en este artículo no profundiza en este ámbito. Sin embargo, dada la envergadura de las mismas, especialmente respecto a centros financieros internacionales, y de su relevancia en términos de potencial impacto, es un área que merece mayor atención. Aunque la información proporcionada por las Cuentas Financieras Nacionales es limitada (p. ej., no permiten distinguir el sector de contraparte

\footnotetext{
7 Un ejemplo es el reciente análisis del Junta Europea de Riesgo Sistémico (JERS) que utiliza datos granulares de tenencias de los sectores financieros de la UE para estimar el impacto que tendría una rebaja masiva de calificación de los emisores situados en el límite inferior del grado de inversión, bajo varios escenarios de distinta severidad (JERS, 2020).
}

cuando son agentes no residentes), las nuevas fuentes de datos permiten análisis más detallados que han de ser explorados.

Otro aspecto relevante que se desprende de nuestro análisis es la creciente relevancia de los sectores no bancarios dentro del sistema financiero español, tendencia que, aunque contenida por el estallido de la crisis sanitaria, hace patente, junto con las consecuencias observadas de esta crisis, la necesidad de progresar en el debate sobre herramientas macroprudenciales para los sectores no bancarios, como diversas voces han remarcado en el seno de la Unión Europea ${ }^{8}$. Los avances de estos sectores apremian tanto para abordar posibles riesgos para la estabilidad financiera generados en ellos, como para evitar que el marco del sector bancario vea reducida su eficacia (p. ej., fruto de arbitraje regulatorio). La envergadura de los flujos transfronterizos también señala la importancia de la cooperación e intercambio de información entre autoridades y jurisdicciones. En este sentido, cabe destacar los trabajos analíticos y desarrollos regulatorios impulsados por el Consejo de Estabilidad Financiera (FSB) en los ámbitos de interconexiones y del sector no bancario. Dado el carácter global del sistema financiero, el análisis de riesgos y el desarrollo de marcos regulatorios requiere también de esa consistencia global.

\section{Referencias bibliográficas}

Aldaroso, I., Huang, W. \& Kemp, E. (2020, September). Cross-border links between banks and non-bank financial institutions. BIS Quarterly Review.

BCE, Banco Central Europeo (2018-2020). Financial Stability Review.

Bricco, J. \& Xu, T. T. (2019). Interconnectedness and Contagion Analysis: A Practical Framework. IMF Working Papers, WP/19/220 IMF.

De Guindos, L. (2018). Coming to the forefront: the rising role of the investment fund sector for financial stability in the euro area.

\footnotetext{
8 Varios altos cargos de instituciones nacionales e internacionales han hecho declaraciones en este sentido en los últimos años (Lane, 2018; Draghi, 2018; De Guindos, 2018).
} 
Draghi, M. (2018). Welcome remarks at the third annual conference of the ESRB.

Financial Stability Board (2011-2020). Global Monitoring Report on Non-Bank Financial Intermediation / Global Shadow Banking Monitoring Report.

Freixas, X., Laeven, L. \& Peydró, J. L. (2015). Systemic Risk, Crises, and Macroprudential Regulation. The MIT Press.
JERS, Junta Europea de Riesgo Sistémico (2016-2020). EU Non-bank Financial Intermediation Risk Monitor / EU Shadow Banking Monitor.

JERS, Junta Europea de Riesgo Sistémico (2020). A system-wide scenario analysis of large-scale corporate bond downgrades.

Lane, P. R. (2018). The Management of Systemic Risks: Current Priorities. 